\title{
TONOMETRIA DA ARTÉRIA FEMORAL EM CÃES SAUDÁVEIS E COM DOENÇA VALVAR MITRAL DEGENERATIVA CRÔNICA
}

\section{TONOMETRY OF THE FEMORAL ARTERY IN HEALTHY DOGS AND IN THOSE WITH CHRONIC DEGENERATIVE MITRAL VALVE DISEASE}

\author{
Rodrigo Bernardes Nogueira ${ }^{1 *}$ \\ Lucas Anacretto Pereira ${ }^{1}$ \\ Luciene Barbosa Gomide ${ }^{1}$ \\ ${ }^{1}$ Universidade Federal de Lavras, Lavras, MG, Brasil \\ "Autor para correspondência - nogueirarb@uol.com.br
}

\begin{abstract}
Resumo
A tonometria de aplanação é um método capaz de aferir a pressão sanguínea dentro de uma artéria. Além de especulações sobre a razão das variações de amplitude das ondas de pulso femoral em cães com doença degenerativa valvar mitral crônica (DVMDC), em medicina, já está demonstrado que muitos sintomas de insuficiência cardíaca em pessoas são atribuíveis a anormalidades funcionais vasculares periféricas e não estão muito associados com parâmetros hemodinâmicos centrais. Desse modo, este trabalho teve por objetivo verificar a aplicabilidade da tonometria vascular em cães, avaliar as medidas de pressão na artéria femoral de cães saudáveis e compará-las com as de cães com regurgitação mitral devido à DVMDC. Os parâmetros tonométricos ainda foram correlacionados com índices cardíacos obtidos pela ecocardiografia. Os dados foram obtidos de 10 cães saudáveis e de 10 cães com DVMDC com regurgitação mitral à ecocardiografia. Observou-se que o período de pré-condução da onda de pulso foi significantemente menor nos animais com DVMDC do que nos saudáveis. Houve uma forte correlação entre a pressão sistólica da artéria femoral com parâmetros determinantes da função sistólica do coração nos animais saudáveis. A tonometria por aplanação da artéria femoral mostrou-se uma técnica aplicável em cães.
\end{abstract}

Palavras-chaves: aplanação; cães; ecocardiografia; vascular.

\begin{abstract}
Applanation tonometry is a method capable of measuring blood pressure in an artery. In addition to speculation about the reason for the variations in amplitude of femoral pulse waves in dogs with chronic degenerative mitral valve disease (CDMVD), in medicine, it has been demonstrated that many symptoms of heart failure in people are attributable to peripheral vascular functional abnormalities, and they are not highly associated with central hemodynamic parameters. Thus, this study aimed to verify the applicability of the vascular tonometry in dogs, to evaluate the pressure measures of the femoral artery in healthy dogs, and compare them with those of dogs with mitral regurgitation due to CDMVD. Moreover, the parameters established for tonometry were correlated with cardiac index obtained by echocardiography. For this, the data were obtained from 10 healthy dogs and 10 dogs with CDMVD with mitral regurgitation on echocardiography. We observed that the pre-conduction period of the femoral pulse wave was significantly lower in CDMVD than in healthy animals. There was a strong correlation between systolic pressure of the femoral artery with determining parameters of cardiac systolic function in healthy animals. The applanation tonometry of the femoral artery was
\end{abstract}


an applicable technique in dogs.

Keywords: applanation; dogs; echocardiography; vascular.

Enviado em: 24 novembro 2014

Aceito em: 10 dezembro 2015

\section{Introdução}

A endocardiose valvar mitral crônica, também denominada DVDMC, é a causa mais comum de insuficiência cardíaca em cães e tem sido responsável por cerca de $75 \%$ a $80 \%$ de todas as enfermidades cardíacas nesta espécie ${ }^{(1)}$. A DVDMC ocorre devido a um espessamento e encurtamento, que podem acompanhar nodulações nos folhetos valvares, com consequente regurgitação mitral. Tais aspectos promovem o comprometimento do débito cardíaco e desencadeiam a ativação orgânica de mecanismos compensatórios por fatores neuro-hormonais. Uma das consequências da ativação crônica de tais fatores é a modificação da fisiologia vascular normal, o que leva a um aumento sobre a carga de ejeção ventricular esquerda e acaba, ao longo do tempo, agravando a regurgitação mitral e favorecendo a manifestação de sinais clínicos de insuficiência cardíaca ${ }^{(2)}$.

Um dos principais métodos de diagnóstico da DVMDC é a ecocardiografia. Este exame, entre outros aspectos, é capaz de verificar alterações valvares e de determinar disfunções ventriculares sistólicas ou diastólicas ${ }^{(3)}$. Entretanto, tal determinação é muito variável em pacientes com DVMDC, sendo que ainda não há consenso entre os investigadores sobre os parâmetros ecográficos centrais que sejam totalmente confiáveis, uma vez que os distúrbios hemodinâmicos resultantes da regurgitação mitral modificam índices fisiológicos determinadores da atividade do coração (p.ex. pré-carga, contratilidade e pós-carga), o que acaba influenciando a avaliação da função cardíaca ${ }^{(4)}$. Paralelamente, em medicina, já está demonstrado que muitos sintomas de insuficiência cardíaca em humanos são atribuíveis, sobretudo, a anormalidades funcionais vasculares periféricas e não estão muito associados com parâmetros hemodinâmicos centrais, obtidos pela ecocardiografia ${ }^{(5-9)}$. Por outro lado, em medicina veterinária está demonstrado, por meio da ultrassonografia duplex Doppler da artéria femoral, que cães com diferentes enfermidades cardíacas, em diferentes fases de insuficiência cardíaca, apresentam significante diminuição no volume de fluxo sanguíneo na artéria femoral ${ }^{(10)}$. Uma pesquisa realizada na Dinamarca relatou a repercussão da DVMDC em diversos graus de gravidade sobre a variação das ondas de pulso arterial periférico em 247 cães ${ }^{(11)}$. Em 26\% destes animais observou-se uma redução de pelo menos $50 \%$ na amplitude da onda pulsátil. Outros $2 \%$ apresentaram ausência de pulso femoral unilateral ou bilateralmente. Concluiu-se que a redução na pulsação sanguínea da artéria femoral foi devida a uma redução regional ou local do fluxo sanguíneo decorrente da DVMDC ${ }^{(11)}$. No entanto, uma das limitações consideradas por tais autores foi que a amplitude pulsátil periférica foi avaliada subjetivamente por meio da palpação da artéria femoral e que outros estudos quantitativos deveriam ser realizados nesse tipo de paciente ${ }^{(11)}$.

A tonometria de aplanação é um método capaz de aferir a pressão sanguínea dentro de uma artéria, a partir da determinação da força necessária para se aplanar a onda de pressão de pulso na parede de tal artéria, de forma semelhante aos instrumentos utilizados para medir a pressão intraocular. Por meio da tonometria arterial, pode-se, então, adquirir medidas de pressão em determinada artéria e alguns dos seus respectivos índices. Em seres humanos, a análise da onda de pulso por meio desta metodologia tem sido reconhecida como uma técnica simples e reproduzível ${ }^{(12)}$ e é amplamente utilizada para avaliar a dinâmica da onda de pulso em diferentes situações clínicas ${ }^{(13)}$. Desse modo, este trabalho teve por objetivo avaliar as medidas de pressão na artéria femoral de cães saudáveis e compará-las com as de cães com regurgitação mitral, devido à DVMDC, por meio da tonometria vascular, para se verificar a aplicabilidade deste método nesta espécie animal. Além disso, os parâmetros estabelecidos com tal método foram correlacionados com índices cardíacos obtidos pela ecocardiografia, para se avaliarem as possíveis associações entre eles, tanto para os animais saudáveis como para aqueles com DVMDC. 


\section{Material e Métodos}

Os dados foram obtidos junto ao serviço de cardiologia, de um Hospital Veterinário de Ensino, de 10 cães saudáveis e de 10 cães com DVMDC apresentando regurgitação mitral à ecocardiografia, conforme as recomendações de Atkins et al. ${ }^{(14)}$. Os animais dos dois grupos foram submetidos a anamnese, avaliação física, hematológica, eletrocardiográfica e ecocardiográfica. Os cães com arritmias cardíacas patológicas não foram incluídos no experimento. Este estudo advém de um projeto submetido à Comissão Institucional de Bioética para Utilização de Animais da UFLA e aprovado sob número 035/2010.

A frequência cardíaca (FC) foi obtida por eletrocardiografia sincrônica (Pulsepen ${ }^{\circledR}$, DiaTecneMilão,Itália), no momento da realização do exame de tonometria da artéria femoral. A pressão arterial sistólica (PAS), pressão arterial diastólica (PAD) e a pressão arterial média (PAM) foram obtidas pelo método oscilométrico indireto (Monitor DX 2020, Dixtal ${ }^{\circledR}$ ), no membro torácico esquerdo, com o animal em decúbito lateral, seguindo as orientações de Brown \& Henik ${ }^{(15)}$.

O exame ecocardiográfico foi realizado em todos os animais sem qualquer tipo de sedação ou anestesia em modos bidimensional, $\mathrm{M}$, Doppler pulsado (PW), contínuo $(\mathrm{CW})$, por mapeamento de fluxo em cores (CFM) e Doppler tecidual (DT). Todos os parâmetros e índices obtidos pela ecocardiografia seguiram as recomendações de Chetboul e Tissier ${ }^{(3)}$. Foi utilizado um aparelho de ecocardiografia (MyLab 40, Esaote $\left.{ }^{\circledR}\right)$, com transdutores de varredura setorial eletrônica de 3 - $11 \mathrm{MHz}$. Todos os exames foram gravados para posteriores avaliações.

No modo bidimensional foram avaliadas as câmaras cardíacas e, especialmente na visão paraesternal direita transversal, foi mensurado o diâmetro do átrio esquerdo (AE) e a dimensão da raiz aórtica (AO). A fração de ejeção (FE) do ventrículo esquerdo foi calculada pelo método de Simpson, na visão paraesternal direita longitudinal. No modo $\mathrm{M}$, foi calculada a fração de encurtamento (FS), na visão paraesternal direita transversal. No modo Doppler por mapeamento de fluxo em cores, na visão paraesternal esquerda apical quatro câmaras, foi calculada a fração regurgitante mitral (FRM) para dentro do átrio esquerdo, pela relação entre a área do AE e da FRM. Ainda na mesma visão, foram observadas as ondas de fluxo mitral (onda E e onda A) e ao modo Doppler tecidual foram mensuradas as ondas Em e Am do segmento septal do ventrículo esquerdo ${ }^{(16)}$. Três medidas consecutivas de cada variável foram obtidas e o valor médio foi calculado. A partir dos valores médios foram estabelecidas as seguintes relações dentre as variáveis ecocardiográficas anteriormente avaliadas: AE/AO, E/A, E/ $\mathrm{Em}, \mathrm{Em} / \mathrm{Am}$.

A onda de pulso femoral foi registrada por tonometria de aplanação sobre a artéria femoral direita. Para análise por tonometria de aplanação da artéria femoral, cada paciente foi colocado em ambiente tranquilo, confortável, em decúbito lateral direito e submetido a um repouso até que se tranquilizasse. Em seguida, as ondas de pulso na porção mais proximal possível da artéria femoral direita foram palpadas. Após a localização por palpação da artéria femoral em sua origem pélvica, o transdutor mensurador de pressão do aparelho de tonometria (sensor do tonômetro) foi mantido sobre tal local, em posição vertical em relação à artéria femoral. As medidas tonométricas foram então efetuadas e registradas em software próprio do equipamento de tonometria (Pulsepen ${ }^{\circledR}$, DiaTecne-Milão, Itália). A onda de pulso femoral foi calibrada por meio do uso da PAS e da PAD ${ }^{(17)}$, obtidas conforme descrito anteriormente. Foram obtidos os seguintes parâmetros do software de tonometria da artéria femoral: pressão arterial sistólica (PASf), pressão arterial diastólica (PADf), pressão de pulso (PPf) $=[\mathrm{PASf}-$ PADf], índice de pressão de pulso (IPPf) pela fórmula PPf/PAM, duração da onda de pulso (HPf) e o período de pré-condução (R-fw), que representa o período de tempo entre o pico da onda R no ECG e o início da onda de pulso femoral. Os valores médios de cada um destes parâmetros foram calculados com a média dos registros das ondas de pulso femorais de ao menos 10 ciclos cardíacos (Figura1). A análise estatística foi realizada utilizando-se o software SPSS 17.0, Microsoft Windows. A análise de distribuição da normalidade das variáveis foi realizada pelo teste de Shapiro-Wilk. A comparação entre as medidas das variáveis decorrentes da frequência cardíaca, pressão arterial sistêmica, exame ecocardiográfico e da tonometria da artéria femoral, dos cães saudáveis e dos cães com DVMDC, foi então realizada pelo teste $\mathrm{T}$ de amostras independentes. As associações entre as diferentes variáveis 
estudadas foram analisadas pelo estudo de correlação de Pearson. Um valor de $p<0,05$ foi utilizado para a determinação de significância estatística.
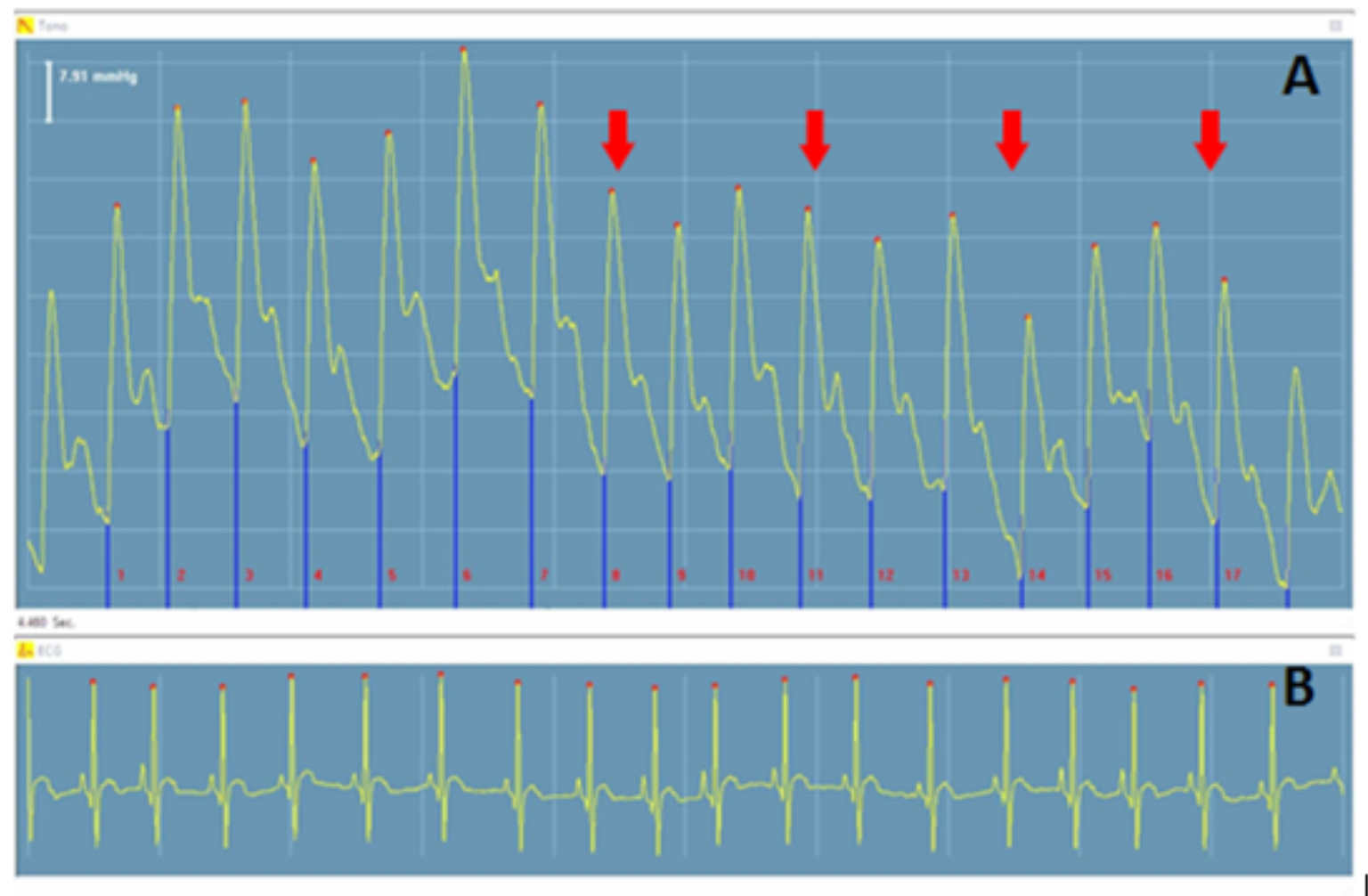

Figura 1: Tonometria de aplanação da artéria femoral direita de um cão saudável. A, Representação da morfologia das ondas de pulso da artéria femoral (setas vermelhas), com a escala da medida de pressão em tal artéria (7,91 mmHg; canto superior esquerdo). B, Traçado eletrocardiográfico sincrônico (Pulsepen ${ }^{\star}$, DiaTecne, Itália).

\section{Resultados}

As raças incluídas no estudo foram Maltês $(n=4)$, Poodle miniatura $(n=14)$, Yorkshire terrier $(n=1)$ e sem padrão racial $(n=1)$. Todos os animais saudáveis eram fêmeas $(n=10)$ e daqueles com DVMDC, seis eram fêmeas e quatro, machos $(n=10)$. Os animais $(n=20)$ não eram castrados. $O$ peso e a idade dos animais saudáveis foram 4,19 $\pm 0,83 \mathrm{~kg}$ de peso vivo e $6,18 \pm 4,63$ anos, e dos animais com DVMDC foram 4,33 $\pm 1,12 \mathrm{~kg}$ e 11,30 $\pm 3,40$ anos, respectivamente. Nenhum animal apresentou características corpóreas compatíveis com obesidade.

No grupo de animais com DVMDC, no exame físico, foi possível auscultar sopro sistólico em foco de valva mitral de grau I/VI em um animal, de grau II/VI em um animal, de grau III/VI em dois animais, de grau IV/VI em quatro animais e de grau VI/VI em dois animais. A ecocardiografia demonstrou espessamento e irregularidades de proporções variáveis, principalmente no folheto valvar mitral anterior, em todos os animais com DVMDC. Em tais animais, no modo Doppler em cores, foi possível avaliar um fluxo regurgitante, na sístole, dentro do átrio esquerdo, de dimensões variáveis em cada animal. Dos cães com DVMDC, seis se encontravam no estágio B1 e quatro em estágio B2, segundo a classificação de Atkins et al. ${ }^{(14)}$. Dos 10 animais enfermos selecionados, quatro estavam sob tratamento com diurético (furosemida) associado a um inibidor da enzima conversora de angiotensina (benazepril). Os demais animais não recebiam qualquer tipo de terapia. 
As medidas das variáveis quantitativas (frequência cardíaca, pressão arterial sistêmica, medidas ecocardiográficas e da tonometria da artéria femoral) dos animais saudáveis e com DVMDC e a respectiva significância estatística na comparação entre elas, estão apresentadas na Tabela 1. As variáveis em que se observou diferença significativa entre os animais saudáveis $(n=10)$ e com DVMDC $(n=10)$ foram FS $(p=0,014)$ e $R-f w(p=0,015)$.

Tabela 1: Comparação estatística das variáveis [média \pm desvio padrão (mín - máx)] determinadas pelos diferentes métodos de avaliação (frequência cardíaca, pressão arterial sistêmica, exame ecocardiográfico e tonometria da artéria femoral) entre os animais saudáveis $(n=10)$ e com DVMDC $(\mathrm{n}=10)$

\begin{tabular}{|c|c|c|c|}
\hline Variáveis & Saudáveis & DVMDC & Significância \\
\hline $\mathrm{FC} \mathrm{(bat} / \mathrm{min})$ & $\begin{array}{c}106,88 \pm 4,97 \\
(102,00-118,00)\end{array}$ & $\begin{array}{c}123,70 \pm 23,02 \\
(91,00-157,00)\end{array}$ & $\mathrm{p}=0,514$ \\
\hline PAS $(\mathrm{mm} \mathrm{Hg})$ & $\begin{array}{c}128,00 \pm 18,15 \\
(103,00-149,00)\end{array}$ & $\begin{array}{c}133,14 \pm 12,64 \\
(115,00-146,00)\end{array}$ & $\mathrm{p}=0,353$ \\
\hline $\mathrm{PAD}(\mathrm{mm} \mathrm{Hg})$ & $\begin{array}{c}84,71 \pm 12,39 \\
(61,00-95,00)\end{array}$ & $\begin{array}{c}90,29 \pm 10,33 \\
(78,00-107,00)\end{array}$ & $p=0,646$ \\
\hline PAM (mm Hg) & $\begin{array}{c}101,71 \pm 17,09 \\
(72,00-117,00)\end{array}$ & $\begin{array}{c}105,57 \pm 10,95 \\
(90,00-119,00)\end{array}$ & $\mathrm{p}=0,241$ \\
\hline $\mathrm{AE} / \mathrm{AO}$ & $\begin{array}{c}1,38 \pm 0,12 \\
(1,20-1,54)\end{array}$ & $\begin{array}{c}1,72 \pm 0,41 \\
(1,25-2,76)\end{array}$ & $\mathrm{p}=0,162$ \\
\hline $\mathrm{E} / \mathrm{A}$ & $\begin{array}{c}1,50 \pm 0,43 \\
(0,63-2,05)\end{array}$ & $\begin{array}{c}1,52 \pm 0,69 \\
(0,76-2,73)\end{array}$ & $p=0,056$ \\
\hline $\mathrm{E} / \mathrm{Em}$ & $\begin{array}{c}10,30 \pm 3,61 \\
(7,50-17,75)\end{array}$ & $\begin{array}{c}11,20 \pm 2,68 \\
(7,36-15,50)\end{array}$ & $\mathrm{p}=0,477$ \\
\hline $\mathrm{Em} / \mathrm{Am}$ & $\begin{array}{c}0,92 \pm 0,48 \\
(0,46-1,64)\end{array}$ & $\begin{array}{c}1,05 \pm 0,62 \\
(0,63-2,71)\end{array}$ & $\mathrm{p}=0,947$ \\
\hline $\mathrm{FE}(\%)$ & $\begin{array}{c}63,12 \pm 8,79 \\
(53,00-75,00)\end{array}$ & $\begin{array}{c}71,00 \pm 6,21 \\
(61,00-77,00)\end{array}$ & $\mathrm{p}=0,599$ \\
\hline FS (\%) & $\begin{array}{l}44,98 \pm 10,83 * \\
(30,00-58,00)\end{array}$ & $\begin{array}{c}54,47 \pm 4,24 * \\
(44,70-59,00)\end{array}$ & $p=0,014$ \\
\hline FRM (\%) & $\begin{array}{c}46,74 \pm 17,10 \\
(23,07-75,09)\end{array}$ & & \\
\hline PASf (mm Hg) & $\begin{array}{c}117,75 \pm 20,69 \\
(87,00-147,00)\end{array}$ & $\begin{array}{c}129,50 \pm 15,97 \\
(107,00-150,00)\end{array}$ & $\mathrm{p}=0,329$ \\
\hline PADf (mm Hg) & $\begin{array}{c}82,00 \pm 12,01 \\
(61,00-97,00)\end{array}$ & $\begin{array}{c}89 \pm 11,48 \\
(69,00-106,00)\end{array}$ & $\mathrm{p}=0,804$ \\
\hline $\mathrm{PPf}(\mathrm{mm} \mathrm{Hg})$ & $\begin{array}{c}39,75 \pm 12,46 \\
(23,00-66,00)\end{array}$ & $\begin{array}{c}40,90 \pm 11,89 \\
(21,00-62,00)\end{array}$ & $\mathrm{p}=0,802$ \\
\hline IPPf & $\begin{array}{c}0,42 \pm 0,01 \\
(0,27-0,64)\end{array}$ & $\begin{array}{c}0,39 \pm 0,11 \\
(0,22-0,57)\end{array}$ & $\mathrm{p}=0,505$ \\
\hline R-fw (ms) & $\begin{array}{c}83,00 \pm 23,96^{*} \\
(44,00-114,00)\end{array}$ & $\begin{array}{c}77,60 \pm 8,97^{*} \\
(61,00-91,00)\end{array}$ & $\mathrm{p}=0,015$ \\
\hline $\mathrm{HPf}(\mathrm{ms})$ & $\begin{array}{c}498,75 \pm 86,64 \\
(350,00-592,00)\end{array}$ & $\begin{array}{c}531,50 \pm 90,60 \\
(385,00-670,00)\end{array}$ & $\mathrm{p}=0,995$ \\
\hline
\end{tabular}

FC, frequência cardiaca; PAS, pressão arterial sistólica; PAD, pressão arterial diastólica; PAM, pressão arterial média; $\mathbf{A E}$, diâmetro do átrio esquerdo; $\mathbf{A O}$, diâmetro da raiz aórtica; $\mathbf{E}$, velocidade do fluxo sanguíneo mitral recente; $\mathbf{A}$, velocidade do fluxo sanguíneo mitral tardio; $\mathbf{E m}$, velocidade do miocárdio na diástole recente; Am, velocidade do miocárdio na diástole tardia; FE, fração de ejeção; FS, fração de encurtamento; FRM, fração regurgitante mitral; PASf, pressão femoral sistólica; PADf, pressão femoral diastólica; PPf, pressão de pulso femoral; IPPf, índice de pressão de pulso femoral; R-fw, periodo de pré-condução; HPf, duração da onda de pulso.* significante 
Três animais avaliados não foram incluídos no estudo, sendo substituídos, devido à presença de tremores musculares em membros pélvicos, na região da artéria femoral. Estes tremores estavam relacionados à ansiedade e comprometeram a evidência das ondas de pulso obtidas por meio do transdutor no software de tonometria.

O estudo de correlação mostrou elevada associação positiva no grupo de animais saudáveis entre a PASf e a FS (r=0,819; $\mathrm{p}=0,013)$, a PADf e a FS $(r=0,718 ; \mathrm{p}=0,045)$ e a PASf e a FE $(r=0,713 ; \mathrm{p}=0,047)$. As pressões arteriais sistêmicas não apresentaram correlação com as demais variáveis estudadas nos animais saudáveis ou com DVMDC. Nos animais com DVMDC, observou-se que a variável obtida pela tonometria R-fw apresentou correlação positiva com o índice $\mathrm{E} / \mathrm{Em}$ da ecocardiografia $(\mathrm{r}=0,663$; $\mathrm{p}=0,037)$. A PPf apresentou associação negativa com a idade $(\mathrm{r}=-0,647 ; \mathrm{p}=0,043)$ e positiva com $\mathrm{o}$ índice Em/Am da ecocardiografia $(\mathrm{r}=0,710 ; \mathrm{p}=0,022)$. Para as demais variáveis estudadas não se observaram correlações significativas.

\section{Discussão}

Neste trabalho, objetivou-se avaliar a utilização da tonometria vascular para verificar a sua aplicabilidade em cães, a relação de suas medidas com índices cardíacos centrais e as possíveis diferenças nas medidas de pressão da artéria femoral em cães saudáveis e com DVMDC. O estudo mostrou que a tonometria vascular é um método simples, indireto, indolor e capaz de obter as ondas de pressão na artéria femoral de cães. Observou-se que a PASf apresentou associação significativa e elevada com a FS e com a FE da ecocardiografia. A PADf também apresentou alta correlação com a FS. Na tonometria da artéria femoral o parâmetro que apresentou significância entre os animais saudáveis e com DVMDC foi o R-fw. Em seres humanos, estudos semelhantes utilizando a tonometria da artéria femoral em pacientes com regurgitação mitral ainda não foram realizados; portanto, não se realizou uma confrontação com estes resultados obtidos em cães. Entretanto, a discussão dos resultados aqui obtidos foi considerada a seguir associando-os aos aspectos fisiopatológicos da DVMDC em cães. O presente estudo é o primeiro a verificar a aplicabilidade da tonometria vascular na clínica veterinária, tanto em animais saudáveis quanto em pacientes com DVMDC. A caracterização dos animais saudáveis e com DVMDC neste estudo se deu inicialmente por meio da história clínica e da auscultação cardíaca no exame físico. A presença de sopro sistólico em foco de mitral, associada com a determinação de regurgitação valvar mitral e da relação $\mathrm{AE} / \mathrm{AO}$ por meio da ecocardiografia, permitiram a definição do estado de saúde dos animais, seguindo as recomendações de Atkins et al. ${ }^{(14)}$. Apesar da relação $\mathrm{AE} / \mathrm{AO}$ não ter apresentado diferença significativa entre os animais saudáveis e com DVMDC, os valores médios de tal relação, assim como os seus limites máximos, foram maiores nos animais com DVMDC do que nos saudáveis. Este aspecto corrobora com a literatura ${ }^{(14)}$, sugerindo o aumento da pressão e no tamanho do átrio esquerdo devido à regurgitação mitral evidenciada em alguns animais com DVMDC, em relação aos saudáveis. A maioria dos animais com DVMDC foi classificada em estágio B1 e o restante em estágio B2. Considerando que o avanço do estágio da DVMDC está associado à intensificação dos sinais clínicos de insuficiência cardíaca ${ }^{(17)}$, os parâmetros relacionados à tonometria da artéria femoral poderiam apresentar diferenças entre os estágios da DVMDC. No entanto, observou-se que os índices ecocardiográficos E/A, E/Em, Em/Am, e FE nos animais com DVMDC não apresentaram diferença significativa em relação aos dos animais saudáveis, mas os seus valores médios e seus limites foram compatíveis com disfunção cardíaca nos portadores de $\operatorname{DVMDC}^{(3)}$.

AFRM apresentou um percentual de regurgitação mitral que variou de discreto a moderado nos animais com DVMDC, compatível com os estágios mais iniciais da doença ${ }^{(3)}$. A FS foi significativamente maior nos animais com DVMDC do que nos animais saudáveis. Este resultado também está comumente associado com a DVMDC em fase inicial em cães, devido à diminuição relativa da pós-carga, associada à manutenção da função sistólica ventricular esquerda ${ }^{(18 ; 3)}$. A FC, apesar de não apresentar diferença significativa entre saudáveis e com DVMDC, apresentou os valores médios e o limite máximo, muito maiores nos animais com DVMDC, do que nos animais saudáveis. Este achado está em acordo com a literatura ${ }^{(2)}$, uma vez que pôde sugerir que a ativação simpática esteve presente nos animais com regurgitação mitral, o que é comum em cães com DVMDC em fase inicial ${ }^{(2)}$. 
Paralelamente, apesar de se encontrarem os valores próximos aos limites fisiológicos de normalidade, as pressões arteriais sistêmicas (PAS, PAD e PAM), embora não tenham sido significantemente diferentes entre os animais saudáveis e com DVMDC, apresentaram, de modo semelhante à FC, valores médios superiores nos cães com DVMDC, o que também sugere que fatores simpáticos e neuro-hormonais possivelmente estavam intensificados neste grupo de animais, conforme observado por Oyama ${ }^{(2)}$. Estes achados são importantes, pois a ativação neuro-hormonal tem ação sobre a dinâmica arterial periférica ${ }^{(18)}$, o que pode modificar a pressão sanguínea na artéria femoral nos animais com DVMDC. Isto pôde ser observado no presente estudo, pois as variáveis obtidas pela tonometria da artéria femoral (PASf, PADf e PPf), apesar de não apresentarem diferenças significativas entre os animais saudáveis e com DVMDC, tiveram os valores médios maiores nos animais com DVMDC, que nos saudáveis. Este aspecto permite considerar que, talvez, se o número de animais observados por grupo no presente estudo fosse maior, diferenças estatísticas significativas estariam presentes. Como a pressão arterial é dependente da resistência periférica e do débito cardíaco ${ }^{(18)}$, pode ser que a resistência, dependente da vasoconstrição da artéria femoral e das arteríolas periféricas, decorrente da ativação neuro-hormonal, tenha influenciado tais índices de pressão (PASf, PADf e PPf), aumentando os seus valores nos animais com DVMDC ${ }^{(2)}$. Esta hipótese é considerada tendo em vista que o débito cardíaco em animais com DVMDC é menor ${ }^{(3)}$, o que, sem os efeitos da ativação neuro-hormonal, deveria baixar e não aumentar as medidas de pressão sanguínea na artéria femoral nestes animais.

Outras medidas obtidas neste estudo relacionadas a tonometria da artéria femoral foram a PPf e o IPPf. As duas medidas também não apresentaram diferença significativa entre os animais saudáveis e com DVMDC. A variação dos valores médios da PPf e do IPPf também foi sutil entre os cães saudáveis e com DVMDC e tais variáveis não se mostraram sensíveis para a diferenciação da condição clínica destes animais, no presente estudo. Esta variação mínima nos valores médios da PPf e do IPPf talvez reflita os resultados encontrados nos índices ecocardiográficos E/A, E/Em, Em/Am, e FE nos animais com DVMDC, que não foram significativamente diferentes em relação aos dos animais saudáveis. Por outro lado, a PPf apresentou associação significante e negativa com a idade nos animais com DVMDC. A pressão de pulso é o resultado da contração cardíaca e das propriedades da circulação arterial, representando o componente pulsátil da pressão arterial, sendo influenciada também pela fração de ejeção, rigidez das artérias, redução precoce da onda de pulso e pela frequência cardíaca ${ }^{(18)}$. Destes fatores, em seres humanos, o aumento da rigidez arterial decorrente da idade normalmente está associado com variações nas pressões de pulso ${ }^{(18)}$. Este pode ter sido um aspecto relacionado à idade dos animais estudados, que influenciou a PPf no presente trabalho. Outro aspecto a ser considerado é que um estudo observou que o volume de fluxo sanguíneo da artéria femoral nos cães velhos foi menor que nos cães jovens ${ }^{(19)}$. Este aspecto pode ter influenciado a PPf em função da idade nos animais aqui estudados; no entanto, é difícil afirmar tal hipótese com os resultados aqui apresentados. Deste modo, outros experimentos deveriam ser delineados para verificar a importância da idade sobre a pulsação arterial periférica em cães.

A HPf, que representa a duração da onda pulsátil na artéria femoral, apesar de não apresentar diferenças significativas entre animais saudáveis e com DVMDC, apresentou os valores médios maiores nestes últimos. Desse modo, a onda de pulso foi mais rápida nos animais saudáveis do que nos com DVMDC. Assim, pode-se dizer, pelo fato de a HPf ser uma medida de tempo, que uma das possíveis influências sobre a mesma seria a variação da FC, que teria uma relação inversa com a HPf. No entanto, além de a FC não ter apresentado correlação com a HPf, o valor médio da FC foi mais elevado nos animais com DVMDC que apresentaram o HPf mais duradouro, se comparado com o dos animais saudáveis. Desse modo, pode-se especular que algum outro fator, como o comprometimento do débito cardíaco consequente da regurgitação mitral nos animais com DVMDC, pode ter promovido um menor volume sanguíneo circulante na artéria femoral, como observado por Nogueira et al. ${ }^{(20)}$ em diferentes enfermidades cardíacas, gerando uma pulsação de menor volume sanguíneo, porém de maior duração periférica, por modificações na dinâmica vascular normal. De qualquer forma, esta hipótese deveria ser comprovada por meio de mais estudos.

Por fim, no presente trabalho, a única variável que se diferenciou significantemente na comparação entre os animais saudáveis e os animais com DVDMC, dentre todas as variáveis avaliadas pela tonometria da artéria femoral, foi o R-fw, que representa o período de pré-condução, entre o início da sístole e o aparecimento da onda de pulso femoral. Considerando-se que o R-fw também é uma 
medida de tempo calculada a partir da onda $\mathrm{R}$ do eletrocardiograma até o início da onda de pulso, sugere-se que seja dependente das variações da FC. No presente estudo, a FC foi maior nos doentes do que nos saudáveis, o que pode ter influenciado de fato o resultado do R-fw, diminuindo a sua duração nos doentes. Por outro lado, o R-fw não apresentou correlação com a FC, nem no grupo dos animais saudáveis, nem dos doentes, para sustentar tal hipótese. O R-fw apresentou correlação positiva com o índice EEm da ecocardiografia nos animais doentes. Na ecocardiografia, o EEm é um índice que auxilia na determinação de insuficiência cardíaca em casos de DVMDC, por estar relacionado às variações da pressão de enchimento do ventrículo esquerdo ${ }^{(4)}$. Os autores consideram o valor do EEm acima de 13 significante para tal finalidade em cães ${ }^{(4)}$. No presente estudo, os animais com DVMDC apresentaram um valor médio de 11,20 de EEm. Apesar de este valor não ser compatível com sobrecarga de pressão no ventrículo esquerdo e consequente insuficiência cardíaca congestiva, segundo os critérios de Schober et al. ${ }^{(4)}$, pode-se especular que o R-fw pode ter sofrido influência da variação de tal pressão ventricular nos animais com DVMDC e ser um sinalizador periférico de tal alteração. Entretanto, uma avaliação mais detalhada com tal variável deveria ser realizada para se aprimorar tais considerações.

Além da correlação EEm com R-fw nos animais com DVMDC, nos animais saudáveis houve correlação significante da variável ecocardiográfica FS com PASf e PADf da tonometria, assim como da FE com a PASf. Estas associações foram interessantes, pois sugerem que os índices ecocardiográficos de avaliação da função sistólica do ventrículo esquerdo podem ter forte relação com as medidas de pressão sanguínea obtidas na artéria femoral, por meio da tonometria. Em pessoas, um estudo comprovou que um índice da tonometria da artéria radial, dependente da pressão e do tempo, pode ser utilizado como um critério de avaliação de performance sistólica do ventrículo esquerdo ${ }^{(21)}$. As variáveis relacionadas à pressão arterial sistêmica (PAS, PAD e PAM) não apresentaram correlação com as mesmas variáveis ecocardiográficas. Em seres humanos, está comprovado que a PAS é muito mais elevada em artérias periféricas (p.ex. artéria braquial) do que nas artérias mais centrais ${ }^{(22)}$. Este aspecto pode justificar a falta de correlação das variáveis ecocardiográficas centrais com a PAS sistêmica nos animais saudáveis. No entanto, a PASf e a PADf mantiveram alta associação com a FS e a FE, sugerindo serem medidas tonométricas da artéria femoral, que merecem mais estudos relacionados aos parâmetros ecocardiográficos determinantes da performance cardíaca. Vale salientar que tais correlações não foram observadas nos animais com DVMDC. Isto pode ter ocorrido devido à interferência de disfunções cardíacas decorrentes da DVMDC e/ou da consequente ativação neurohormonal sistêmica, que pode ter influenciado a associação de tais medidas nestes pacientes.

Dentre as limitações do presente estudo, pode-se considerar que, apesar de todos os animais com DVMDC apresentarem regurgitação mitral, os mesmos estavam em estágio inicial (B1 e B2) e talvez estudos com animais em estágios mais avançados ou já com sinais clínicos de insuficiência cardíaca possam trazer influências mais evidentes sobre as medidas tonométricas da artéria femoral em pacientes com tal enfermidade. Além disso, alguns dos animais com regurgitação mitral do presente estudo estavam sob terapia médica, o que pode ter interferido nas respectivas medidas tonométricas, devido a influência terapêutica direta ou indireta sobre a dinâmica arterial ${ }^{(1 ; 14)}$.Um outro aspecto que também deve ser mencionado é o fato de este trabalho ter avaliado apenas a artéria femoral direita, desconsiderando os possíveis efeitos pressóricos sobre a mesma artéria contralateral. Além disso, a determinação da ativação neuro-hormonal poderia ser mais bem avaliada por meio da mensuração de peptídeos vasoativos séricos, assim como a sua correlação com as variáveis centrais e periféricas, fatos que podem ser considerados em trabalhos futuros. Como limitação, também deve ser mencionado que, apesar da percepção das ondas de pulso da artéria femoral ser um pouco mais sutis na avaliação física dos cães com DVMDC em relação aos saudáveis, a avaliação por palpação das ondas pulsáteis da artéria femoral não foi realizada com critérios neste trabalho devido ao caráter subjetivo e imensurável de precisão, se comparado ao aspecto quantitativo determinado pela tonometria arterial, no presente estudo.

Umas das dificuldades enfrentadas no desenvolvimento experimental, responsável pela exclusão de três cães deste estudo, foi a interferência por tremores musculares na região de avaliação da artéria femoral dos animais sobre a obtenção de um traçado estável das ondas pressóricas obtidas pelo transdutor de tonometria. Também deve ser considerado que o transdutor da tonometria deve permanecer o mais perpendicular possível sobre o vaso avaliado e estar gentilmente posicionado, sem 
grande compressão no vaso.

Finalmente, a mensuração das ondas de pressão e a obtenção de seus índices a partir da artéria femoral dos animais em estudo foi realizada de maneira fácil e indolor. Estudos mais aprofundados em diversas enfermidades da clínica veterinária deveriam ser mais realizados com a tonometria vascular, tendo em vista que é um método considerado preciso e exequível de se obter conhecimento sobre a dinâmica vascular periférica que, em muitas situações, pode servir para o melhor entendimento da modificação da pós-carga ventricular e da ação de hipotensores.

\section{Conclusões}

Como conclusão, pode-se dizer que, no presente trabalho, a tonometria da artéria femoral mostrou-se um método aplicável em cães. Tanto a pressão sanguínea sistólica como diastólica, da artéria femoral, não apresentaram diferença entre os animais saudáveis e os com DVMDC. O período de pré-condução da onda de pulso diferenciou os animais com DVMDC dos animais saudáveis. A pressão sistólica da artéria femoral pareceu ser um bom critério periférico de avaliação da performance sistólica do ventrículo esquerdo nos animais saudáveis e merece mais estudos. Serão necessários mais estudos para que a técnica seja aplicada com segurança e sensibilidade nos cães normais e portadores de DVMDC.

\section{Agradecimentos}

Agradecemos a Fundação de Apoio a Pesquisa do Estado de Minas Gerais (FAPEMIG) pelo apoio financeiro para o desenvolvimento deste trabalho.

\section{Referências}

1. Hamlin RL. Geriatric heart diseases in dogs. Veterinary Clinics of North America - Small Animal Practice. 2005;35(3):597-615.

2. Oyama MA. Neurohormonal activation in canine degenerative mitral valve disease: implications on pathophysiology and treatment. Journal of Small Animal Practice. 2009;50(1):3-11.

3. Chetboul V, Tissier R. Echocardiographic assessment of canine degenerative mitral valve disease. Journal of Veterinary Cardiology. 2012;14(1):127-48.

4. Schober KE, Hart TM, Stern JA, Li X, Samii VF, Zekas LJ, Scansen BA, Bonagura JD. Detection of congestive heart failure in dogs by Doppler echocardiography. Journal of Veterinary Internal Medicine. 2010;24(6):1358-68.

5. Franciosa JA, Park M, Levine B. Lack of correlation between exercise capacity and indices of left ventricular performance in heart failure. American Journal of Cardiology. 1981;47(1):33-39.

6. Franciosa JA, Leddy CL, Wilen M, Schwartz DE. Relation between hemodynamic and ventilatory response in determining exercise capacity in severe congestive heart failure. American Journal Cardiology. 1984;53(1):127-134.

7. Mancini DM, Lejentel TH, Factor S, Sonnenblick E. Central and peripheral components of cardiac failure. American Journal of Medicine. 1986;80(2):2-12.

8. Wilson JR, Martin JL, Schwartz D, Ferraro N. Exercise intolerance in patients with chronic heart failure. Role of impaired nutritive flow to skeletal muscle. Circulation. 1984;69(6):1079-1087.

9. Wilson JR, Rayos G, Yeoh TK, Gothard P, Bak K. Dissociation between exertional symptoms and circulatory function in patients with heart failure. Circulation. 1995;92(1):47-53.

10. Nogueira RB, Muzzi RAL. Evaluation of aging on muscle blood flow in conscious healthy dogs by duplex Doppler ultrasonography. Veterinary Research Communication. 2010;34(4):307-14.

11. Tarnow I, Olsen LH, Jensen MB, Pedersen KM, Pedersen HD. Determinants of weak femoral artery pulse in dogs with mitral valve prolapse. Research Veterinary Science. 2004;76(2):113-20. 
12.Benetos A, Laurent S, Hoeks AP, Boutouyrie PH, Safar ME Arterial alterations with aging and high blood pressure. A noninvasive study of carotid and femoral arteries. Arteriosclerosis, Thrombosis, and Vascular Biology - Journal of the American Heart Association. 1993;13(1):90-97.

13.Kelly R, Fitchett D. Noninvasive Determination of Aortic Input Impedance and External Left Ventricular Power Output: A Validation and Repeatability Study of a New Technique. Journal of the American College of Cardiology. 1992;20(4):952-963.

14. Atkins C, Bonagura J, Ettinger S, Fox P, Gordon S, Haggstrom J, Hamlin R, Keene B, Luis-Fuentes V, Stepien R. Guidelines for the Diagnosis and Treatment of Canine Chronic Valvular Heart Disease. Journal of Veterinary Internal Medicine. 2009;23(6):1142-1150.

15.Brown SA, Henik RA. Diagnosis and treatment of systemic hypertension. Veterinary Clinics of North America - Small Animal Practice. 1998;28(4):1481-1494.

16. Tidholm A, Ljungvall I, Hoglund AB, Westling AB, Haggstrom J. Tissue Doppler and Strain Imaging in Dogs with Myxomatous Mitral Valve Disease in Different Stages of Congestive Heart Failure. Journal of Veterinary Internal Medicine. 2009;23(6):1197-1207.

17. Salvi P, Lio G, Labat C, Ricci E, Pannier B, Benetos A. Validation of a new non-invasive portable tonometer for determining arterial pressure wave and pulse wave velocity: the Pulse Pen device. Journal Hypertension. 2004;22(12):2285-2293.

18. Wilson JR, Martin JL, Schwartz D, Ferraro N. Exercise intolerance in patients with chronic heart failure. Role of impaired nutritive flow to skeletal muscle. Circulation 1984;69(6):1079-1087.

19.Nogueira RB, Muzzi RAL. Evaluation of aging on muscle blood flow in conscious 32 healthy dogs by duplex Doppler ultrasonography. Veterinary Research Communication. 2010;34(4):307-314.

20.Nogueira RB, Fernández Del Palacio MJ, Talavera J, Muzzi RAL. Alterations in the large peripheral circulation in dogs with heart failure. The Veterinary Journal. 2011;188(1):101-104.

21.Tartiere JM, Logeart D, Beauvais F, Chavelas C, Kesri L, Tabet JY, Cohen-Solal A. Non-invasive radial pulse wave assessment for the evaluation of left ventricular systolic performance in heart failure. European Journal of Heart Failure. 2007;9(5):477-483.

22. Bortolotto LA. Central pressure: how to understand in clinical practice? Revista Brasileira de Hipertensão. 2009;16(1):46-47. 